\title{
Public Procurement of Innovation Through Increased Startup Participation: The Case of Digipolis (Research-in-progress)
}

\author{
Ben De Coninck \\ Royal Military Academy / \\ KU Leuven \\ ben.deconinck@mil.be
}

\author{
Stijn Viaene \\ Vlerick Business School / \\ KU Leuven \\ stijn.viaene@vlerick.com
}

\author{
Jan Leysen \\ Royal Military Academy \\ jan.leysen@mil.be
}

\begin{abstract}
Previous research has identified numerous obstacles that hinder the efficient procurement of innovation by the public sector. This paper introduces the case of Digipolis - the public ICT service provider of the City of Antwerp in Belgium. In 2015, the company implemented a comprehensive overhaul of its procurement strategy centered around 3 key components: a flexible procurement process, a community built around Digital Antwerp, and a challenge-oriented company culture. The case adopts a holistic perspective on the implementation of innovation procurement in a local public sector organization, and investigates the specific conditions and mechanisms that allowed to leverage the Antwerp startup community in order to increase the number of purchased innovative solutions. The case also sheds light on how public procurement of innovation can lead to knowledge-intensive entrepreneurship - an area that is still largely undiscovered.
\end{abstract}

\section{Introduction}

The primary objective of public procurement is to allow a public organization to obtain products, consisting of goods, services, or combinations of the two [1]. Repeatedly, however, governments have turned to public procurement as an instrument to realize horizontal policy objectives, such as sustainability, social inclusion, and employment generation $[2 ; 3]$. The incorporation of so-called 'secondary' objectives is motivated by the pervasive impact of public procurement on the European Union's domestic economy, as indicated by its 14 percent share of the region's annual Gross Domestic Product [4].

One such policy objective that has gained a renewed interest in the European context in recent years is the use of public procurement as a vehicle to encourage innovation, commonly referred to as Public Procurement of Innovation (PPI) [5; 6]. The motivation to do so is frequently cited as aiming to improve the quality and efficiency of public services, while simultaneously encouraging innovation in companies and having the public sector act as a launching customer for these innovative solutions [2; $4 ; 8 ; 10]$.

Despite the interest from various government levels, a 2015 report from the European Research Area and Innovation Committee identified an underexploited opportunity to spur innovation using procurement in Europe [8]. Reasons for this failure to capture the full potential of innovation procurement can be the barriers to effective implementation that previous research has uncovered [e.g., 11; 12; 13]. Examples include low capabilities of procurers, a lack of close and early supplier engagement, narrowly-defined tender specifications, and contract size constraints. Such barriers impact how and under what conditions public procurement can stimulate innovation and achieve innovative outputs [11]. Startups and Small and Medium-sized Enterprises (SMEs), having limited access to resources and capabilities, perceive some of these obstacles as more burdensome, limiting their participation in public sector procurement [14].

In this research-in-progress we introduce the case of Digipolis - the public, not-for-profit ICT service provider for the various public sector organizations in the City of Antwerp, Belgium. In 2015, Digipolis realized that in order to keep up with the fast pace of technological innovation, it needed to radically overhaul its conventional procurement strategy. Rather than consistently turning towards a handful of traditional ICT vendors, Digipolis aimed to attract small-scale innovative companies. In cooperation with local partners, the company implemented a comprehensive overhaul of its procurement strategy, thereby boosting the number of startups that take part 
and increasing the procurement of innovative solutions.

The case contributes to the existing literature in that it identifies the specific conditions and mechanisms that allowed Digipolis to repeatedly purchase innovative solutions. The identification of such levers has been cited as needing further investigation [11].

Additionally, Georghiou et al. [6] underscore the importance of purchasing organizations, which may often be at sub-national level, taking ownership of innovation procurement. On a related note, many of the cases in the literature describe individual PPI projects [e.g., $1 ; 13 ; 15 ; 16 ; 17]$. This case, however, adopts a more holistic perspective on the implementation of innovation procurement compared to other cases, and demonstrates how such ownership was taken by a local public sector organization.

Lastly, the case sheds light on how public procurement of innovation can lead to knowledgeintensive entrepreneurship. Timmermans and ZabalaIturriagagoitia [14] rightly highlighted the lack of academic attention paid to this topic. That is, many cases investigate the promotion of innovative behavior among existing firms rather than looking into the entrepreneurial opportunities associated with PPI.

The main purpose of our empirical case study is to understand $(i)$ what possible key components of an public innovation procurement strategy can be, (ii) the barriers and pitfalls that can arise when implementing such a strategy, and (iii) the main outcomes of the revised way-of-working.

\section{Related Literature}

Public procurement of innovation has been defined by Edquist et al. [18] as "when a public agency acts to purchase, or place an order for, a product [...] that does not yet exist, but which could probably be developed within a reasonable period of time, based on additional or new innovative work by the organization(s) undertaking to produce, supply and sell the product being purchased". This definition emphasizes that some innovation must occur before the product can be delivered $[1 ; 16]$.

Some authors have warned of a bias towards radical innovation by defining PPI as the purchase of a product "that does not yet exist" [2; 11; 14]. Indeed, innovation can take on many forms and does not necessarily require the development of a completely new-to-the-world product $[1 ; 2]$. Additionally, such an interpretation tends to overlook the impact of so-called innovation-friendly (or innovation-enhancing) procurement $\quad-$ which underscores that public procurement is first and foremost aimed at solving a public sector need but that, where possible, innovation should be allowed and encouraged as a secondary objective (a "byproduct") [2; 19]. This broader understanding of public procurement fades the strict divide between procurement of innovation and regular procurement. It also has powerful implications for practitioners as it acknowledges that every procurer has a role to play in realizing innovation impacts [2].

Innovation scholars generally agree with the claims made by policy makers that public procurement can have a positive impact on innovation $[13 ; 23]$. However, given the complex nature of the public sector, consisting of a broad spectrum of different types of organizations, we introduce Uyarra \& Flanagan's product-based typology of public procurement [2].

The authors distinguish two principal dimensions of products, namely "type of market" (i.e., whether the products are generic or dedicated) and "type of production system" (i.e., whether the products are specialized or standardized). The result is a four-fold typology of public procurement. The authors go on to argue that each market/production system combination requires a different set of "procurement practices" (or "barriers" when phrased in a negative manner) in order to foster innovation.

Other studies have found comparable barriers to public procurement of innovation [e.g., 11; 12;13]. One such fundamental barrier that is frequently identified in the academic literature is the lack of competences on the part of the procurer $[6 ; 11 ; 14]$. Innovation procurement distinguishes itself from regular procurement in that it involves a different level of complexity, and thus requires a unique combination of knowledge, organizational arrangements, and job structuring [12]. Particularly in the case of $R \& D$-intensive products, a lack of capabilities at lower levels of governance - such as local administrations - has been found to restrict efficient procurement of innovative solutions [11]. Aho et al. [24] introduced the concept of an "intelligent customer" in this regard to denote a customer that takes "actions to develop a cohort of trained professionals and to support them through networks to exchange ideas and raise skills".

Another notable barrier to effective procurement of innovative solutions is the issue of overlyperspective tender specifications, limiting the freedom of the supplying company to suggest unsolicited, out-of-the-box solutions $[2 ; 11 ; 12]$. In the case of innovation procurement, specifications phrased in terms of functions or outcomes have been 
shown to be more fitting $[11 ; 12 ; 17]$. Letting go of these strict specifications requires close and early supplier engagement in order to further an environment of mutual trust, which allows to reduce the transactions costs by stimulating the relationship to evolve from a traditional arm's-length transaction towards a partnership $[2 ; 11 ; 12 ; 17]$.

A topic still largely undiscovered in the academic literature is to what extent public procurement of innovation can lead to knowledge-intensive entrepreneurship (KIE) [14]. Timmermans and Zabala-Iturriagagoitia distinguish between a direct influence, where PPI influences entrepreneurship directly by providing market opportunities, and an indirect influence, where entrepreneurship is indirectly stimulated by providing technological opportunities. The authors claim that PPI can stimulate KIE by providing entrepreneurial opportunities that can lead to the creation of new entrepreneurial ventures.

\section{Research Design}

To conduct the current research, we applied a qualitative methodology centered around an explanatory single case study in order to better understand the specific conditions and mechanisms that allowed Digipolis to repeatedly purchase innovative solutions. Case studies, seeking to understand phenomena in their naturally-occurring setting, allow researchers to provide description, build theory, or test theory [20;22]. They have been identified as an ideal method to generate managerially-relevant knowledge $[21 ; 22]$.

Our research focuses on the events that transpired over the time period January 2015 to July 2017. We investigate the initial launch of the new procurement strategy and broaden the scope to include developments after the strategy was implemented.

Data was gathered through telephone, and in some cases face-to-face, interviews; internal Digipolis documents; tender calls; and secondary data from news and/or web archives. Three semistructured interviews of around one hour each were conducted with the Chief Executive Officer (CEO) of Digipolis. Questions were prepared beforehand, but the open-ended nature allowed to make use of additional insights provided by the interviewee. The first interview focused on obtaining a broad understanding of the company's old and new procurement strategy, whereas the second and third interview delved deeper into key aspects, such as the specifics of the new procurement procedure.
Additionally, four semi-structured interviews, around fifty minutes each, were conducted with four suppliers of Digipolis: NSX (interviewee: Business Development Manager), Hifluence (Partner), Sirus (Chief Technology Officer), and Delaware Consulting (Sales Manager). These interviews focused on the supplier's evaluation of the procurement strategy in order to provide a comprehensive description. All interviews were conducted over the six-month period of March 2017 to August 2017, and were fully transcribed.

Data from the interviews was complemented with documents and secondary data. The documents consisted primarily of internal Digipolis presentations on its procurement strategy and on specific projects, as well as an in-depth tender call to better understand how the company approaches startups. Blog posts and news articles helped to gain more insight into the new way-of-working, and assisted in reconstructing timelines when necessary.

\section{Case Study}

Digipolis is the public, not-for-profit ICT service provider of the various public sector organizations in the City of Antwerp, Belgium. As the IT partner of the city, Digipolis aims to support Antwerp in its ambition to offer comprehensive digital services to residents, businesses, visitors, students, and so on. To this end, the ICT service provider is responsible to meet the wide array of digital needs of the city administration, the local police and fire brigade, the local social welfare organization, and various public schools in Antwerp. Digipolis offers its customers the Antwerp public sector - extensive solutions, including the development of software, the implementation of hardware and infrastructure, the coaching of end users, and the incorporation of the solution in the organization.

At the start of 2015, Digipolis asked itself the question how it can provide better software solutions to the City of Antwerp. Top management realized that because it was frequently partnering with traditional, large-scale software vendors, such as IBM, Oracle and SAP, the company was missing out on innovative solutions typically developed by startups. Given the presence of a sizeable startup community in Antwerp, leveraging their knowledge and innovativeness presented great opportunities. The CEO, Peter Crombecq, recounted:

"Technological innovation moves at lightning speed. We felt that we needed to create an environment that places creativity and innovation at its center, with a 
primary role for small-scale, innovative companies, citizens and school communities."

-Chief Executive Officer (Digipolis)

A closer investigation of why Digipolis was failing to attract startups revealed a procurement process dictated by the needs and wishes of largescale ICT vendors. A gap existed between the supply and demand-side, as exemplified by a lack of close and early supplier engagement. Rather than jointly designing a solution to Antwerp's digital needs, lengthy tender documents were published that specified in great detail the desired solutions, leaving little to no room for creativity on the part of the supplying company. Such calls for tenders were typically published on the national e-Procurement platform, which startups in Belgium have identified as being rather rigid and difficult to navigate. The procedures itself were lengthy, ranging from four months up to two years, requiring potential suppliers to run through a wide array of administrative steps and documents. Additionally, the size and duration of a typical contract was large and spanning across an extended period of time; it commonly involved the delivery of end-to-end solutions. As such, Intellectual Property Rights (IPR) were usually not discussed. IPR remained at the large-scale ICT vendor, while Digipolis obtained licensing rights. As a final step in the procurement procedure, contracts were awarded based on three key criteria: price, quality, and timing of delivery. Extra criteria could be taken into account on a case-by-case basis. Essentially, the bureaucratic procedures required a sizeable up-front investment from companies in terms of time and resources with only a small chance of being awarded the contract. As recognized by one supplier:

"We normally do not bid on government contracts. Just reading through the tender documents often already takes a couple of days. As a small company we do not have those resources..."

-Chief Technology Officer (Sirus)

In January 2015, Digipolis embarked on a strategic journey aimed at implementing a new procurement strategy in order to radically boost the startup participation rate and increase the number of purchased innovative solutions. The objective 'from 0 to 100 startups in just six months' represented a clear break with the past. From January to May 2015, the company set up an internal co-creation exercise, inviting in-house employees to brainstorm about what a renewed procurement process could look like. While employees were given the freedom to make unconventional suggestions, the internally-focused effort proved to be more difficult than expected: neither employees nor top management were familiar with the needs and wants of a startup company. To resolve the deadlock, local partners were brought in to help design a strategy tailored to startups. The Advisory Board consisted of iMinds (a Flemish ICT research institute), Startups.be (a national interest group for Belgian startups), and the Startup Manager of the City of Antwerp. Jointly, the team developed a new procurement strategy built around 3 key components: a flexible procurement process, a community built around Digital Antwerp, and a challenge-oriented company culture.

\subsection{Component 1: A Flexible FAST Procurement Procedure}

To design the new procurement procedure, Digipolis chose a radical approach. By starting from a blank canvas, the organization aimed to ensure that every step in the new way-of-working functioned as a catalyst - rather than a hindrance - to attract innovative, small-scale companies. The new procedure was dubbed "FAST", referring to the drastic decrease in throughput time from four months (at minimum) to four weeks.

The FAST procedure divides the procurement process into four consecutive steps, starting with the publishing of a challenge. The idea behind challenges is to let go of the preconception that the contracting authority is the expert.

"Analysts in Digipolis were traditionally trained to write extensive tender specification documents outlining the desired solution in detail. But this undermines innovation... Who knows, maybe other experts know better than us?"

-Chief Executive Officer (Digipolis)

Instead of Digipolis designing the solution to the digital need of the client, the ICT service provider publishes a problem statement on its online website in order to challenge companies to come up with a solution themselves. In doing so, Digipolis aims to preserve the creative freedom of the supplying company, which no longer has to adhere to the narrowly-defined performance, technical, and functional specifications put forward by Digipolis experts. This prevents limiting itself to the knowledge and expertise that is available in-house, stimulating the development of out-of-the-box solutions provided by small-scale, innovative companies. 
Additionally, the concept of challenges emphasizes the need for lightweight tender specification documents. In the past, analysts often recycled templates, leading to lengthy documents containing overly-prescriptive specifications and a lot of legal jargon. Out of the forty to fifty pages that a typical tender document counted, only a minority outlined useful information that contributed to the development of a solution. To halt this practice, the company made a conscious choice to adopt short, bite-sized tender documents, ensuring that every section contains relevant information and is phrased in a 'sexy manner'.

In a next step, interested companies are invited to present their proposed solution during a thirty-minute pitch followed by a fifteen-minute Q\&A session. The pitch resolves around the key question "why are you the best fit for the job?", and should outline both the key business and technical aspects of the proposal. The jury consists of three members of the public sector client and two technically-focused Digipolis employees. As such, not only buyer-supplier interaction is stimulated, but also direct contact between the end client and the supplier, creating an opportunity for co-creation between the supply and demand-side before an official tender is submitted. By evaluating the concept of the proposed solution and the skills and experience of the company, the jury selects the three to five best candidates, who are invited to move to the next step. Although pricebased criteria come into play in a later phase, the initial selection is focused on value-based metrics only.

The best candidates are asked to submit a tender, which is again presented in front of a jury during a one to two hour presentation. This time, however, the presentation should include both the business and technical aspects, as well provide an in-depth answer to the award criteria. The official tender can take on the form of the slideshow used during the presentation. Rather than requiring extensive tenders backed with a considerable number of bureaucratic attachments (e.g. certificates), companies are asked the bare minimum in order to minimize the up-front time and resource investment.

Submitted tenders are evaluated based on four criteria in order to award the contract to one supplier: (i) the solution concept (i.e. quality, availability, and usability of the solution), (ii) the technical specifications and the innovativeness of the proposed solution, (iii) the sprint planning for development with monthly releases, and (iv) the pricing model, including possible commercialization opportunities. The criteria go beyond solely price-based metrics and underscore Digipolis' commitment to support the growth of startups. Instead of blindly claiming exclusivity over the developed solutions, Digipolis is open to talk about the commercialization of the product by the startup outside the Antwerp public sector. To this end, IPR are negotiated on a case-bycase basis together with the supplier. Additionally, the evaluation criteria emphasize the need for monthly releases of 'shippable products' throughout the development process, tying into an agile approach that is typically preferred by startups. Agile development also ensures close, iterative interaction between the supplier, Digipolis, and the end client throughout the development process, furthering the relationship that was started during the initial pitch.

The no-nonsense way-of-working during the FAST procedure has resulted in a considerable decrease in the time required to award contracts. Whereas old procedures took four months to two years depending on the amount of the contract, the new procedure takes on average four weeks. Furthermore, by drastically reducing the administrative burden on the bidding companies, the up-front investment in the pre-award phase has gone down, allowing easier access to resource-constrained startups.

\subsection{Component 2: A Community Around Digital Antwerp}

The ambition of Digipolis to support the City of Antwerp is broader than merely adopting a FAST procurement procedure. Parallel to the development of the new process, the ICT service provider took steps towards setting up a community of startups centered around "building Digital Antwerp together". The home base of the community is the DigAnt Café group that Digipolis created on the social networking website Meetup.com. It is influenced by the Quadruple Helix Innovation Model, and brings together employees from the public and private sector, academics, and all types of enthusiasts such as students and hobbyists [27].

The open community serves two main purposes. First, it is aimed at attracting and identifying creative entrepreneurs that can help to solve the public sector challenges facing Digipolis as an ICT service provider. As such, the collaborative and interactive nature of the community introduces startups to Digipolis as a potential public sector client, and acts as a launching board for future challenges. One supplier noted:

"Without the DigAnt Café, it is likely that we would have never participated in challenges. I went to one 
of the meetups and started talking with some of the people. For us, that was the point at which we decided to give it a try."

\section{-Chief Technology Officer (Sirus)}

Second, the DigAnt community is used as a broader platform for knowledge sharing, networking, and gaining inspiration. To this end, regular meetups, focusing on hot topics such as blockchain and Internet of Things, are organized in the form of talks, hackathons, and testimonials.

It is important to note the interplay that may arise between these two primary objectives of the community. An interesting example is the search for potentially interesting applications of blockchain technology in Antwerp. In December 2016, Digipolis organized an informative meetup during which several experts were invited to shed light on the what, how, and why questions surrounding this upcoming technology [28; 29]. Over a hundred community members showed interest for the event, allowing Digipolis to communicate to a wide audience the city's ambition to implement this innovative technology in future projects. As such, the meetup served as the kickoff of two separate avenues investigating blockchain in a government context $-\mathrm{a}$ first in Belgium.

On the one hand, four challenges were launched in February 2017, requesting the development of Proof-Of-Concepts (POCs) that investigate in-depth the use of blockchain technology to aid in the city administration [9]. These challenges involved a cross-organizational cooperation with representatives of the Flemish government, the Federal government, and the Flemish organization for IT-ers of local authorities (V-ICT-OR) [30]. Eventually, 32 organizations submitted 109 proposals. The success was, among others, due to the upfront signaling of future needs during the preceding meetup.

On the other hand, Digipolis launched the 'Blockchain for Antwerp' competition in April 2017. The competition was aimed at supporting Antwerp's journey towards becoming a Smart City. More specifically, a challenge was launched on the Digipolis website inviting anyone to suggest " $a$ creative, comprehensible, and realistic concept that implements blockchain technology in a Smart City environment." [9] Again, the initial meetup had served as a prior communication of future demand. In this case, however, the community was also leveraged as a way to evaluate the submitted concepts. To this end, two meetups were organized in June 2017 during which the submitting startups pitched their concept in front of the audience, who decided the top-3 $[31 ; 32 ; 33]$. The winning idea -
Synd-e-cus, a blockchain application that supports owners in shared buildings with the maintenance and other shared responsibilities - has been added to the longlist of solutions to be potentially implemented in the context of Antwerp's Smart City program in 2018 [33].

The search for the potential of blockchain technology in Antwerp is exemplary of the collaborative nature of the new procurement strategy - in which Digipolis provides an open knowledgesharing platform to anyone that is interested, and which, in turn, feeds back into better quality solutions to the challenges facing the Antwerp public sector.

\subsection{Component 3: A Challenge-Oriented Company Culture}

A final key component of Digpolis' new procurement strategy is the fundamental shift in company culture towards an open, entrepreneurial mindset that places at its center thinking in terms of challenges rather than solutions.

The first challenge that was launched following the procedural overhaul exemplified the need for such an organizational culture shift. The challenge, inviting companies to suggest solutions that would alleviate Antwerp's mobility problems, was awarded to the concept 'SlimNaarAntwerpen.be' - an online website that provides the fastest route and mode of transport in Antwerp, taking into account road constructions, traffic jams, and other obstacles. The startup that had suggested the idea, Engine27, was still in a 'pre-startup phase' at the time of participating in the challenge. It had not been founded yet; the idea only existed in the mind of its (future) founder. For Digipolis employees, accustomed to working together with traditional ICT vendors, this represented a clear break with the past. Engine27 has since grown into a company specializing in the integration of backend systems, custom software development, and business intelligence [35].

In order to successfully collaborate with startups, Digipolis needed to evolve from an internallyfocused ICT service provider that was tightly in control of the procurement outcomes towards a company that recognizes itself as part of a broader innovation network. To this end, a variety of info sessions, workshops, and bootcamps were organized to get everyone in the company involved.

It is interesting to note that, rather than being a one-off investment, change management remains an ongoing process in Digipolis. To stimulate employees to adopt an entrepreneurial mindset, the ICT service 
provider still regularly organizes bootcamps. The exercises during the bootcamps are aimed at putting employees in the shoes of startups in order to better understand how they experience the procurement process, and at encouraging employees to think in terms of challenges rather than designing the solutions themselves.

Digipolis' community approach to procurement is tightly related to the change management process. For example, startups are encouraged to work from the Digipolis offices, accelerating the internal culture shift:

"By working alongside startups on a daily basis, our employees are infected with their entrepreneurial and creative mentality. It helped tremendously to change the culture [...] towards a more open-minded agile attitude."

\section{-Chief Executive Officer (Digipolis)}

\subsection{Enabler of Change: Antwerp City Platform as a Service}

Simultaneous to its strategic transformation in 2015, Digipolis started with the development of Antwerp City Platform as a Service (ACPaaS). ACPaaS refers to the local ICT architecture of the City of Antwerp that consists of a collection of small, reusable engines. An engine is a specific limited functionality that can be called upon as a service using an Application Programming Interface (API). On top of the ACPaaS, new software applications can be developed that link back to the underlying infrastructure engines using APIs. To give one example, when developing a new application, a developer can simply call upon the existing payment engine rather than implementing the process for a payment transaction from scratch. The value of ACPaaS lies in the fact that it creates a plug-and-play environment of functional building blocks that can be used during application development.

The start of ACPaaS was an important facilitator to jumpstart the changes in the procurement strategy in 2015. It prevented the development of new software applications, which is outsourced to startups, from taking on unwieldy proportions. Without the ACPaaS environment, the project scope of new apps would likely only have been manageable by traditional, large-scale ICT vendors, defeating the very purpose of the new way-of-working. Additionally, ACPaaS imposed challenge-oriented thinking on the business analysts as they were suddenly faced with an architectural vision that was novel in the context of the Antwerp (and even
Belgian) public sector. In this sense, the innovative architectural platform also served as a clear signal towards the startup community that Digipolis was serious about its new procurement strategy, and that it was open to out-of-the-box ideas.

Progressively, the procurement strategy has evolved beyond the development of the ACPaaS environment. In fact, over the two-year period July 2015-2017, nearly twice as much challenges have been launched that were unrelated to ACPaaS (42 challenges vs. 72 challenges). A key underlying driver is the company's deliberate strategy to downsize software purchases as much as possible, away from large-scale, monolithic solutions.

\section{Results}

Since the launch in June 2015, the new procurement strategy has resulted in a number of tangible and intangible benefits. Firstly, the interplay between the three key components has allowed Digipolis to drastically increase the startup participation rate in Antwerp public ICT procurement. Prior to 2015, the service provider had never worked with a startup; it consistently turned to traditional ICT vendors to satisfy the digital needs of customers. However, just two years after the strategic overhaul, around half of the city's annual $€ 8$ million ICT budget is allocated to buying from startups. On average, the company has launched one challenge per week, with 412 startups performing 553 pitches throughout 114 challenges over the two-year period June 2015-2017.

This influx of startups has provided Digipolis with a rich collection of innovative ideas that can be transformed into tangible solutions, allowing Digipolis to become one of the frontrunners in the digitalization of the Belgian public sector. Prime examples are the search for applications of blockchain technology in a government context and the development of the ACPaaS environment. By publishing such progressive challenges, Digipolis is able to better support Antwerp in its aim of becoming the digital capital of Flanders.

Adoption of the community approach has led to around 550 creative entrepreneurs subscribing to the online website to keep themselves informed on the latest Digipolis challenges and news, helping to boost the average number of companies competing for a contract from $1-5$ to $10-15$. Feeding into this is the DigAnt Café community, counting just over 1600 members, that have gathered around 28 themed meetups so far. One of the primary intangible outcomes of the collaborative nature of the new 
procurement strategy has been the organizational culture shift away from a closed, self-centered point of view.

A fourth notable benefit resulting from the strategic transformation is the realization of efficiency gains. The FAST procedure has resulted in a drastic decrease in the procedural throughput time from four months (at minimum) to four weeks. As such, speed has become one of the dominant KPIs for the company - speed in publishing, speed in contracting, and speed in delivery. The enhanced efficiency is also characterized by a drastic decrease in bureaucratic overhead as less time and effort is required in every step of the procurement process.

Startups emphasize the role of Digipolis as a stepping stone for future growth, providing valuable learning opportunities that can be leveraged both in the public and private sector. One startup commented:

"It is a strong concept to support startup growth. Rather than receiving impersonal subsidies, startups gain hands-on experience and obtain a solid reference as stepping stone to other public and private sector customers."

\section{-Business Development Manager (NSX)}

Tightly related to this aspect is the positioning of Digipolis as an innovation-oriented ICT service provider. The company's collaborative approach to ICT procurement have allowed it to become an attractive partner of the Antwerp startup community.

A third aspect that is highly valued by startup suppliers is the accessible nature of the FAST procurement procedure. The combination of a transparent, non-bureaucratic process with a low throughput time ensures a limited upfront investment in the pre-award phase. A supplier phrased it as follows:

"One of the reasons why we keep returning to Digipolis is because it is easy. Their procedures do not leave us feeling frustrated; everything works smoothly."

\section{-Partner (Hifluence)}

Regardless of these beneficial effects, it is important to note that, as with many culture shifts, also Digipolis faced the challenge of general inertia associated with organizational change. Shifting the company culture towards a challenge-oriented mindset demands that business analysists, who are typically trained to analyze a problem and develop a solution, let go of the preconception that they are the expert. Instead, their function evolves towards identifying an unmet need, translating this future demand in terms of a challenge that appeals to creative entrepreneurs, and using the co-creation opportunities in the procurement process to work alongside startups. This requires a radically different skillset and vocabulary than what they are used to. Both startups and Digipolis agree that the realization of this 'coworking-with-startups' mentality remains an ongoing process. Startups described the example of how some pitches still revolve too much around the supplier delivering a one-sided presentation of the proposed solution, rather than this moment of interaction consistently being used to enter into a dialogue between the demand and supply-side. This is one of the reasons why two years after the strategic transformation Digipolis continues to organize bootcamps for its employees.

Other challenges that have been identified by startups relate to difficulties in resource planning that originate from working in a fixed price/variable scope environment. As common in public procurement contracts, the budget allocated to the development of IT applications is determined upfront. However, the agile way-of-working adopted under the new procurement strategy emphasizes the need for iterative development cycles, allowing to gain new insights with each cycle. Many startups, lacking experience in project management, have the tendency to easily accept extra functionality being added to the scope of the project. This, however, squeezes their profit margins, making it more difficult to reach the breakeven point at the end of the project. In a sense, it can be argued that this is an issue inherent to agile software development in many public sector contexts, stemming from public procurement legislation that restricts pricing opportunities.

Finally, startups have also indicated difficulties with finding the right balance between highlighting the primary business insights and highlighting the main technical aspects of the proposed solution during the thirty-minute pitch. Evidently, it is important not to confuse the public sector client by going too technical, but at the same time the technically-focused Digipolis jury members have to be convinced of the feasibility of the solution. However, both the supply and demand-side agree that standardizing the interaction during the pitch to aid in this balancing act would do more harm than good.

\section{Discussion and Conclusion}

This research-in-progress has introduced the case of Digipolis - the public ICT service provider for the 
various public sector organizations in the City of Antwerp, Belgium. We started in 2015 when Digipolis embarked on its journey to implement a procurement strategy overhaul aimed at leveraging the Antwerp startup community in order to increase the number of purchased innovative solutions.

The case has demonstrated the importance of letting go of the preconception that the contracting authority is the expert. Instead, challenges aim to safeguard the creative freedom of the supplying companies, thereby stimulating the development of out-of-the-box solutions. The concept of challenges is in line with the idea of functional or outcome-based specifications that have been described in the literature as an important practice that can drive innovation. Additionally, in this case, management also decided to adopt challenge-oriented thinking as part of the organizational culture. This corresponds to what Uyarra \& Flanagan [2] described as "innovation-friendly procurement", where innovation is allowed and encouraged as a by-product whenever possible. This fades the strict divide between procurement of innovation and regular procurement. It also has powerful implications for practitioners as it acknowledges that every procurer has a role to play in realizing innovation impacts.

In Uyarra \& Flanagan's four-fold typology [2], the case of Digipolis corresponds to "experimental procurement", meaning that it involves the procurement of adapted technical (software) solutions, where the supply of inputs comes from a "community of specialists" (i.e., the startup community) and the clients' demands are precise and heterogenous (i.e., the Antwerp public sector). In such an experimental setting, the public sector acts as an experimental or lead user. Digipolis takes on this experimental role by being the frontrunner in the digitalization of the Belgian public sector. Two prime examples are the development of the ACPaaS environment, and the search for applications of blockchain technology. Taking the example of blockchain, the four contracted POCs may eventually lead to software solutions that are commercialized both in and outside of the Antwerp public sector.

The case also provides an interesting example of how close and early buyer-supplier interaction, frequently identified in the literature as an important lever for innovation procurement, can take place. The DigAnt community approach shows how the community can be leveraged to identify, attract, and engage with creative entrepreneurs in early stages. The meetups have also been used as a means to signal future demand upfront, allowing suppliers to gear up for supply.
The case contributes to the existing literature in that, rather than addressing the broad question whether public procurement can stimulate innovation, it focuses on the specific conditions and mechanisms that allowed Digipolis to repeatedly purchase innovative solutions. The identification of such levers has been cited as needing further investigation [11].

Additionally, Georghiou et al. [6] emphasize the multi-level governance that characterizes successful public innovation procurement. The authors underscore the importance of purchasing organizations, which may often be at sub-national level, taking ownership of innovation procurement. This case outlines how Digipolis implemented a comprehensive overhaul of its procurement strategy in order to leverage the innovativeness of the startup community. As such, it adopts a holistic perspective on the implementation of innovation procurement, and demonstrates how such ownership was taken by a local public sector organization.

Lastly, the case sheds light on how public procurement of innovation can lead to knowledgeintensive entrepreneurship. Timmermans and ZabalaIturriagagoitia [14] rightly highlighted the lack of academic attention paid to this topic. The anecdotal example of Engine27 shows how Digipolis created an entrepreneurial market opportunity (a "direct influence"), leading to the startup being founded. It can also be argued that the development of ACPaaS has led to the emergence of technological opportunities ("indirect influence"), which may contribute to providing entrepreneurial opportunities.

\section{References}

[1] Edquist, C., \& Zabala-Iturriagagoitia, J.M. (2012). "Public Procurement For Innovation As Mission-Oriented Innovation Policy”. Research Policy, 41(10), 1757-1769.

[2] Uyarra, E., \& Flanagan, K. (2010). "Understanding The Innovation Impacts Of Public Procurement". European Planning Studies, 18(1), 123-143.

[3] McCrudden, C. (2004). "Using Public Procurement To Achieve Social Outcomes". Natural Resources Forum, 28, 257-267.

[4] Schwimann, I. (2016). "Speech At The Fifth Annual Conference On Innovative Public Procurement". Retrieved May 20, 2017, from http://ec.europa.eu/growth/toolsdatabases/newsroom/cf/itemdetail.cfm?item_id=8866.

[5] Edler, J., \& Georghiou, L. (2007). "Public Procurement And Innovation-Resurrecting The Demand Side". Research Policy, 36(7), 949-963.

[6] Georghiou, L., Edler, J., Uyarra, E., \& Yeow, J. (2014). "Policy Instruments For Public Procurement Of Innovation: 
Choice, Design And Assessment". Technological Forecasting \& Social Change, 86, 1-12.

[7] European Commission. (2014). "Innovation Procurement". Retrieved July 15, 2017, from https://ec.europa.eu/digital-single-market/en/innovationprocurement.

[8] ERAC. (2015). "ERAC Opinion On Innovation Procurement". ERAC Secretariat, Brussels.

[9] Digipolis Antwerpen. (s.d.). "Opdrachten en Uitdagingen". Retrieved August 15, 2017, from https://antwerpen.digipolis.be/nl/opdrachten.

[10] European Commission. (2015). "Workshop: Legal Framework For Innovation Procurement". Retrieved July 15, 2017, from https://ec.europa.eu/digital-singlemarket/en/news/workshop-legal-framework-innovationprocurement.

[11] Uyarra, E., Edler, J., Garcia-Estevez, J., Georghiou, L., \& Yeow, J. (2014). "Barriers To Innovation Through Public Procurement: A Supplier Perspective". Technovation, 34(10), 631-645.

[12] Wilkinson, R., Georghiou, L., Cave, J., et al. (2005). "Public Procurement For Research And Innovation: Developing Procurement Practices Favourable To R\&D And Innovation". Office for Official Publications of the European Communities, Luxembourg.

[13] Rolfstam, M. (2009). "Public Procurement As An Innovation Policy Tool: The Role Of Institutions". Science and Public Policy, 36(5), 349-360.

[14] Timmermans, B., \& Zabala-Iturriagagoitia, J.M. (2013). "Coordinated Unbundling: A Way To Stimulate Entrepreneurship Through Public Procurement For Innovation". Science and Public Policy, 40, 674-685.

[15] Edquist, C., \& Zabala-Iturriagagoitia, J.M. (2015).

"Pre-Commercial Procurement: A Demand Or Supply Policy Instrument In Relation To Innovation?”. R\&D Management, 45(2), 147-160.

[16] Edler, J., Ruhland, S., Hafner, S., Rigby, J., Georghiou, L., Hommen, L., Rolfstam, M., Edquist, C., Tsipouri, L., \& Papadakou, M. (2006). "Innovation And Public Procurement: Review Of Issues At Stake". Fraunhofer Institute for Systems and Innovation Research, Karlsruhe.

[17] Yeow, J., Uyarra, E., \& Gee, S. (2011). "Sustainable Innovation Through Public Procurement: The Case Of 'Closed Loop' Recycled Paper'. Manchester Business School Working Paper Number 615, Manchester Business School, Manchester.

[18] Edquist, C., Vonortas, N.S., Zabala-Iturriagagoitia, J.M., \& Edler, J. (2014). "Public Procurement Of Innovation". Edward Elgar Publishing, Cheltenham.

[19] Edquist, C. (2017). "Innovation-Enhancing Public Procurement: Describe functions instead of products!". Retrieved July 15, 2017, from https://rio.jrc.ec.europa.eu/en/policy-support-facility/mleinnovation-procurement.

[20] Benbasat, I., Goldstein, D.K., \& Mead, M. (1987).

"The Case Research Strategy in Studies of Information Systems". MIS Quarterly, 11(3), 369-386.

[21] Gibbert, M., Ruigrok, W., \& Wicki, B. (2008). "What passes as a rigorous case study?". Strategic Management Journal, 29(13), 1465-1474.
[22] Yin, R.K. (2014). "Case Study Research: Design and Methods". Fifth Edition, SAGE Publications, Calif.

[23] Dalpé, R., DeBresson, C. \& Xiaoping, H. (1992). "The Public Sector As First User Of Innovations". Research Policy, 21(3), 251-263.

[24] Aho, E., Cornu, J., Georghiou, L., \& Subirá, A. (2006). "Creating an Innovative Europe". Office for Official Publications of the European Communities, Luxembourg.

[25] Rothwell, R. \& Zegveld, W. (1981). "Government Regulations And Innovation - Industrial Innovation And Public Policy”. In: Rothwell, R. \& Zegveld, W. (Eds.), Industrial Innovation and Public Policy. Frances Pinter, London, 116-147.

[26] Flanders Today. (2015). “Antwerp wins award from Global Entrepreneurship Network". Retrieved July 20, 2017, from http://www.flanderstoday.eu/business/antwerpwins-award-global-entrepreneurship-network.

[27] Parveen, S., Senin, A.A., \& Umar, A. (2015). "Organization Culture and Open Innovation: A Quadruple Helix Open Innovation Model Approach". International Journal of Economics and Financial Issues, 5, 335-342.

[28] Digipolis Antwerpen. (2016). "Blockchain-as-aserv-ice voor Antwerpen?". Retrieved August 15, 2017, from https://www.meetup.com/DigAntCafe/events/235856780/.

[29] Digipolis Antwerpen. (s.d.). "Antwerpen verkent mogelijkheden van Blockchain". Retrieved August 15, 2017 from https://antwerpen.digipolis.be/nl/blog/8279c5f3-5e33-4a1f8c19-1200d01f4f26.

[30] Digipolis Antwerpen. (s.d.). "Blockchain Proof Of Concepts Antwerpen: Unieke samenwerking tussen Federale, Vlaamse en lokale overheden". Retrieved August 15, 2017, from https://antwerpen.digipolis.be/nl/blog/83910fee-6130-41b7a950-0d5c0345c97f.

[31] Digipolis Antwerpen. (2017). "Blockchain for Antwerp wedstrijd - Blockchain \& Smart City". Retrieved August 15, 2017, from https://www.meetup.com/DigAntCafe/events/240186714/.

[32] Stad Antwerpen. (2017). "Blockchaininspiratiemiddag". Retrieved August 15, 2017, from https://www.antwerpen.be/nl/info/57288441b1a8a7a1208b 4fa7/blockchain.

[33] Stad Antwerpen. (2017). "Tweede blockchainsessie: ben jij klaar voor de revolutie?". Retrieved August 15, 2017,

from https://www.antwerpen.be/nl/info/595b4939a677930ed520 7853/tweede-blockchainsessie-ben-jij-klaar-voor-derevolutie.

[34] Engine27. (s.d.). "Engine27". Retrieved August 15, 2017, from http://www.engine27.be/. 